\title{
Intervalley Scattering and the Role of Indirect Band Gap AlAs Barriers: Application to GaAs/AlGaAs Quantum Cascade Lasers
}

\author{
J. MC TAViSH*, Z. IKONIĆ, D. INDJin AND P. HARRISON
}

School of Electronic and Electrical Engineering, University of Leeds Leeds LS2 9JT, U.K

\begin{abstract}
We report on the results of our simulations of $\Gamma-X$ scattering in GaAs/AlGaAs heterostructures, discussing the importance of the mole fraction, doping density, and lattice and electron temperature in determining the scattering rates. We consider three systems, a single quantum well (for the investigation of $\Gamma-X$ scattering), a double quantum well (to compare the $\Gamma-X-G$ and $\Gamma-\Gamma$ scattering rates), and an example of a GaAs/AlGaAs mid-infrared quantum cascade laser. Our simulations suggest that $\Gamma-X$ scattering can be significant at room temperature but falls off rapidly at lower temperatures. One important factor determining the scattering rate is found to be the energy difference between the $\Gamma$ - and $X$-states.
\end{abstract}

PACS numbers: 72.10.-d, 73.21.Fg, 73.63.Hs

\section{Introduction}

The working principles of the resonant-tunnelling (or double-barrier) diode (RTD) structure was predicted by Tsu and Esaki [1] following their pioneering work on superlattices in the late 1960s and early 1970s. Realisation of the device in the form of an AlGaAs layer sandwiched between two GaAs regions was achieved by Chang, et al. [2] soon after. Following the work of Sollner [3] who demonstrated operation in the terahertz region, research interest escalated and room temperature operation was achieved in $1985[4,5]$. Such structures have since been shown to be both of broad physical and of technological interest (Aleshkin et al. [6]).

Mendez et al. [7] reported on electron resonant tunnelling in $\mathrm{Ga}_{0.6} \mathrm{Al}_{0.4} \mathrm{As}^{-}$ GaAs- $\mathrm{Ga}_{0.6} \mathrm{Al}_{0.4}$ As structures via states above the AlGaAs potential barrier and later confirmed that these resonances arose from confined $X$-states in the Brillouin

*corresponding author; e-mail: J.P.Mctavish@ljmu.ac.uk 
zone [8]. The importance of such scattering processes in explaining experimental data was confirmed theoretically in the many-band pseudo-potential model calculations of Marsh [9] and also by Liu [10].

The quantum cascade laser (QCL) is an electrically pumped semiconductor laser that emits in the mid-infrared region of the electromagnetic spectrum and shares many features with RTDs, the most notable being both are semiconductor heterostructure devices. Unlike conventional semiconductor injector lasers that make use of electron-hole recombination, QCLs are unipolar devices that make use of intersubband transitions to generate electromagnetic radiation [11-19].

The first experimental realisation of a QCL was based on an InGaAs/InAlAs heterostructure material system lattice matched to an InP substrate [11]. This system has proven popular and an extensive research effort has resulted in a range of devices that operate over a wide range of wavelengths and temperatures $[12,13]$. However, since QCLs are based upon intersubband transitions, it follows that their operation should be independent of the specific semiconductor material system used $[14,15]$. Consequently, research was undertaken on materials other than InGaAs/InAlAs, and resulted in the demonstration in 1998 by Sirtori et al. [16] of a device based upon GaAs/AlGaAs grown on a GaAs substrate. This material system has attracted much attention recently for a variety of reasons [17-23]: (i) AlGaAs semiconductors are the most technologically well developed of the compound semiconductors; (ii) The GaAs substrate is mechanically more robust than InP making the manufacture of devices easier; (iii) GaAs and AlAs have approximately identical lattice constants, so strain at the interface is minimised; (iv) The possibility of new low-loss AlGaAs wave guide designs; (v) The extension of the operating region to the far infrared.

Although GaAs/AlGaAs QCLs have performed well at low temperatures, their operation at room temperature has been more difficult to achieve. One suggestion to extend the working temperature was to increase the $\mathrm{Al}$ content of the barrier regions, since the higher $\mathrm{Al}$ content increases the band offset between the GaAs well and the AlGaAs barrier, thus improving electron confinement [18]. Subsequently, Page et al. [18] demonstrated a QCL based on an increased Al content, from $33 \%$ in the original design [16] to $45 \%$, reporting a significant increase in the operating temperature.

Because $\mathrm{Al}_{x} \mathrm{Ga}_{1-x} \mathrm{As}$ is an indirect band gap semiconductor for $x \geq 45 \%$, it follows that the conduction band minimum in the barriers is at the $X$ point of the Brillouin zone $[24,25]$ and it becomes important to understand the role of intervalley or $\Gamma-X$ scattering when determining the performance of these GaAs based QCLs [26].

Experimental results for the importance or otherwise of $\Gamma-X$ scattering seem confusing: Some authors claim that $\Gamma-X$ scattering can be appreciable, for example inhibiting lasing in AlGaAs QCLs depending on the relative energies of the $X$ and $\Gamma$ states [27], and strongly influences the $I-V$ characteristics 
(depending on the barrier thickness) [28, 29]. Other authors, however, claim that intervalley scattering is negligible [30] and plays no significant role in the behaviour of QCLs.

It is the purpose of this paper to provide a systematic study of $\Gamma-X$ scattering in $\mathrm{GaAs} / \mathrm{Al}_{x} \mathrm{Ga}_{1-x} \mathrm{As}$ heterostructures, using a single quantum well to determine the importance of well width, molar concentration $x$, (lattice) temperature $T_{\text {latt }}$, and doping density $N_{\mathrm{d}}$ (considering the importance of the Pauli blocking). We also consider the application of an electric field and its effect on the scattering rate. After this we consider a double quantum well to determine the role of intervalley scattering in the tunnelling through single layer heterostructures, i.e. $\Gamma-X-\Gamma$ scattering compared with $\Gamma-\Gamma$ scattering. Finally, we estimate the relative importance of intervalley scattering in a realistic QCL device.

\section{Modelling}

We model intervalley scattering according to the work of Raichev [31], who developed a model of phonon-assisted $\Gamma-X$ transfer in periodic GaAs/AlAs (001)grown heterostructures. The analysis was based on the envelope-function approximation for electrons and an improved description of the phonon spectrum, taking into account the considerable difference in the atomic masses of $\mathrm{Ga}$ and $\mathrm{Al}$. In this model there are two principal mechanisms for intervalley scattering: interlayer $\Gamma-X$ scattering and $\Gamma-X$ mixing.

We follow Raichev's notation and consider a GaAs/AlGaAs superlattice, made up of a GaAs well of width $d_{1}$ and an $\mathrm{Al}_{x} \mathrm{Ga}_{1-x} \mathrm{As}$ barrier of width $d_{2}$, and find the scattering rates for $\Gamma-X$ scattering (using the notation of Raichev) from the $\Gamma$-state $n$ to the $X$-states $n^{\prime}$ as

$$
W_{n}=\frac{1}{N_{\mathrm{d}}(n)} \frac{1}{(2 \pi)^{4}} \frac{1}{d} \sum_{n^{\prime}} \sum_{m} H_{m, n^{\prime}}^{\Gamma X}(m) N I_{n, n^{\prime}}^{\Gamma X}(\Delta \varepsilon),
$$

where $N_{\mathrm{d}}(n)$ is the $\Gamma$ sheet density of the state $n$ (we are not considering selfconsistent solutions yet), $d=d_{1}+d_{2}$ is the length of the unit cell and $m$ labels the region (either well or barrier). The terms $H$ in Eq. (1) involve overlap integrals between the $\Gamma$ - and $X$-state wave functions, $G_{n}^{\Gamma}(z)$ and $G_{n^{\prime}}^{X}(z)$ respectively, and the phonon modes

$$
H_{n, n^{\prime}}^{\Gamma X}=\frac{2 \pi D_{\mathrm{m}}^{2} d}{\omega_{\mathrm{m}} \rho_{\mathrm{m}} d_{m}} \sum_{L=1}^{\infty}\left|\frac{1}{d} \int \mathrm{d} z G_{n}^{\Gamma}(z) \sin \left(\frac{\pi L z}{d_{m}}\right) G_{n^{\prime}}^{X *}(z)\right|^{2},
$$

where the range of integration is taken over the well $(m=1)$ or barrier $(m=2)$ region. In Eq. (2) $D_{\mathrm{m}}$ is the deformation potential constant, $\rho_{\mathrm{m}}$ is the density, and $\omega_{\mathrm{m}}$ is the phonon (angular) frequency for the material. The term $N$ contains information about the effective number of phonons, $N=\frac{1}{\exp \left(\beta \hbar \omega_{\mathrm{m}}\right)-1}+\frac{1}{2} \mp \frac{1}{2}$, and where \pm refers to the emission and absorption of a phonon, respectively, with $\beta=1 / k_{\mathrm{B}} T$. Finally, $I_{n, n^{\prime}}^{\Gamma X}(\Delta \varepsilon)$ is given as

$$
\int_{\boldsymbol{k}} \int \mathrm{d}^{2} \boldsymbol{k} f_{n}^{\Gamma}(\boldsymbol{k}) \int_{\boldsymbol{k}^{\prime}} \int \mathrm{d}^{2} \boldsymbol{k}^{\prime} \delta\left[\varepsilon_{n}^{\Gamma}(\boldsymbol{k})-\varepsilon_{n^{\prime}}^{X}\left(\boldsymbol{k}^{\prime}\right)+\Delta \varepsilon\right],
$$


with $\Delta \varepsilon=\mp \hbar \omega_{\mathrm{m}}$. In Eqs. (1) and (2) $X$ refers to $X_{x}$, and for this exploratory work we ignore the contributions from the $X_{x}, X_{y}$ states, and take the $\Gamma$ distribution function to be the usual Fermi-Dirac distribution. As we will consider carrier densities in typical devices of order $10^{11} \mathrm{~cm}^{-2}$, we initially ignore the Pauli blocking in the final state but do consider its order of magnitude effects briefly to confirm or otherwise this assumption.

We find that we can write the expression for $\Gamma-X$ mixing as

$$
W_{n}=\frac{1}{N_{\mathrm{d}}(n)} \frac{2 \pi \alpha^{2}}{\hbar d^{2}} \frac{1}{(2 \pi)^{2}} \sum_{n^{\prime}}\left|C_{n, n^{\prime}}^{\Gamma X_{z}}\right|^{2} J
$$

where $\alpha$ is a single parameter that describes mixing between the envelope functions at the interfaces. $C_{n, n^{\prime}}^{\Gamma X}$ involves the $\Gamma$ and $X_{z}$ envelope functions at the interfaces and is given as $G_{n^{\prime}}^{\Gamma}\left(-d_{1}\right) G_{n^{\prime}}^{X_{z}^{*}}\left(-d_{1}\right)-G_{n^{\prime}}^{\Gamma_{1}}(0) G_{n^{\prime}}^{X_{z}^{*}}(0)$ and the term $J$ is determined from the result

$$
\int_{\boldsymbol{k}} \int \mathrm{d}^{2} \boldsymbol{k} f_{n}^{\Gamma}(\boldsymbol{k}) \delta\left[\varepsilon_{n}^{\Gamma}(\boldsymbol{k})-\varepsilon_{n^{\prime}}^{X_{z}}(\boldsymbol{k})\right]
$$

Heterostructures consist of alternate layers of dissimilar material so that the mass of the carrier is different in these layers and to solve the Schrödinger equation we follow Raichev's [31] suggestion that it is sufficient to adopt the envelope function approximation $[32,33]$ for each valley for the first order calculations of the scattering rates

$$
\left[-\frac{\hbar^{2}}{2} \frac{\mathrm{d}}{\mathrm{d} z}\left(\frac{1}{m^{*}(z)} \frac{\mathrm{d}}{\mathrm{d} z}\right)+V(z)\right] \psi_{n}(z)=\varepsilon_{n} \psi_{n}(z) .
$$

In Eq. (4) $V(z)$ is the band discontinuity at the well/barrier interface and the integer $n$ labels the subbands.

\section{Results and discussion}

We now present the results of our calculations, using the parameter values for the $D_{\mathrm{m}}$ and $\alpha$ given by Raichev [31]. Results for the variation of the scattering rate with the energy separation, $\Delta \varepsilon$, between the lowest $\Gamma$ level and the lowest $X$ level in a single quantum well are presented in Fig. 1. The data were generated by considering a single quantum well with well of width $d_{1}$ and barrier of width $d_{2}$, keeping $d_{1}$ fixed and varying $d_{2}$. Because of the rapid fall off in scattering rate with (lattice) temperature, $T_{\text {latt }}$, the use of a $\log$ scale is appropriate since otherwise the results for $77 \mathrm{~K}$ and $115 \mathrm{~K}$ would be difficult to discern.

We consider two ranges of well width: $D_{1}: d_{1}=35 \AA$ and $10 \AA<d_{2}<$ $90 \AA$ and $D_{2}: \quad d_{1}=30 \AA$ with $10 \AA<d_{2}<65 \AA$. The differences between the two sets of data show the importance of the localisation of the wave function in the wells (which determine the overlap integrals) and also suggest that any theoretical predictions must be interpreted with care, since the differences between corresponding curves in Fig. 1 can be of the order of a factor three or more in the calculated scattering rates. 


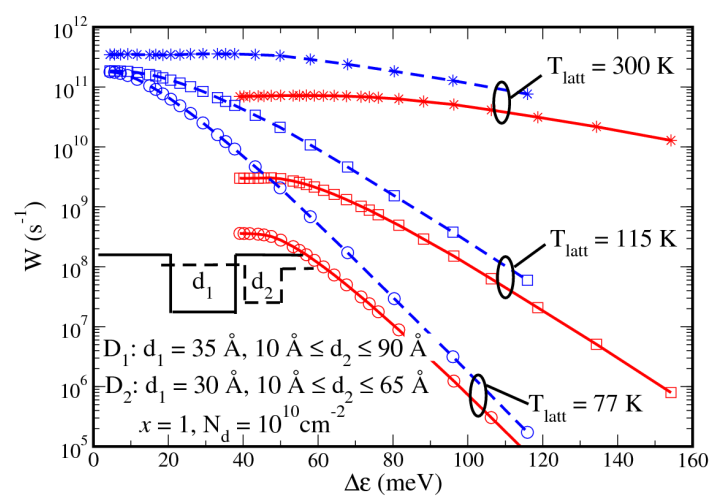

Fig. 1. Scattering rate versus $\Delta \varepsilon$ for a single well for lattice temperatures $77 \mathrm{~K}, 115 \mathrm{~K}$ and $300 \mathrm{~K}$. The dotted curves correspond to the set $D_{1}$, while the full curves correspond to the set $D_{2}$ (see text for explanation). The inset shows the $\Gamma$ (full line) and $X$ (dashed line) potential profiles. Let us note the rapid fall off of the scattering rate with energy and temperature.

From the figure, we have scattering rates $\approx 10^{11} \mathrm{~s}^{-1}$ for room temperature that fall off rapidly with energy difference and with temperature to $\approx 10^{9} \mathrm{~s}^{-1}$. Given that the phonon scattering rates for transitions of interest in realistic devices are typically found to be $\approx 10^{12} \mathrm{~s}^{-1}$ (see later), these results show that $\Gamma-X$ scattering can be important to take into account for devices operating at room temperature.

Many calculations do not distinguish between the different materials that make up the well and boundary regions, and at this point we take advantage of the notation and investigate these differences. We do this by setting the boundary region macroscopic parameters (density and phonon frequency) equal to the values in the well region. We find that there can be differences in the calculated scattering rates of up to around 20-25\%, depending upon the relative sizes of the well and barrier regions and also how different the macroscopic properties between well and barrier are. Thus, for the accurate determination of device operation it is important to consider the effects of both well and barrier materials.

The variation of scattering rate with molar fraction, $x$, is shown in Fig. 2. Here we again consider a single quantum well, with the well width $d_{1}$ taken as $35 \AA$ and the barrier $d_{2}$ as $20 \AA$. The total sheet density $N_{\mathrm{d}}$ is taken as $10^{10} \mathrm{~cm}^{-2}$ and we also investigate the dependence on the lattice temperature and the case of the electron temperature different from the lattice temperature [34], $T_{\mathrm{e}} \neq T_{\text {latt }}$. Again, the results for the lower temperatures are difficult to discern, which necessitates the use of a $\log$ scale.

The results show significant variation with molar fraction, with the scattering rate falling off rapidly with $x$. This variation can be related to the results shown in Fig. 1: as the molar fraction decreases, so the $X$ potential profile rises compared 


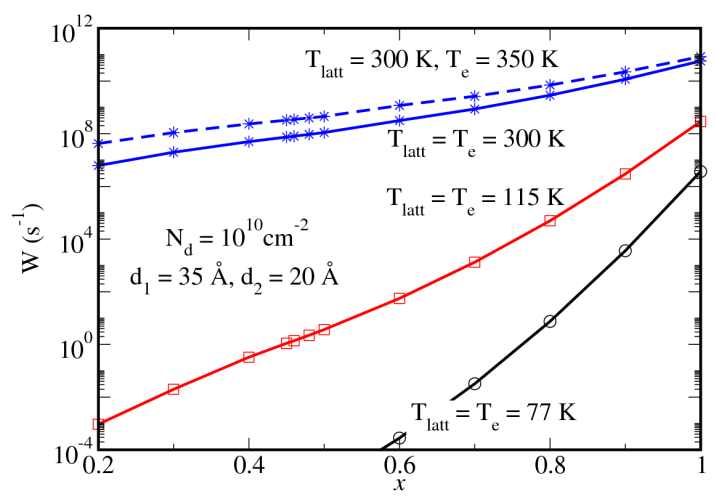

Fig. 2. The variation of the scattering rate with molar fraction, $x$, and lattice temperature, $T_{\text {latt }}$. As the molar fraction gets smaller, the difference between the $\Gamma$ - and $X$-state energies get larger and the scattering rate becomes smaller.

with the $\Gamma$ potential profile, and the energy of the $X$-states rise, implying larger energy differences between the $X$ and $\Gamma$-states and so, from Fig. 1, a smaller scattering rate. Of course, another effect is that as $x$ gets smaller we eventually have no barrier to contend with, and so no $X$ - state.

A modest variation with the electron temperature, $T_{\mathrm{e}}$, is also noted. As the electron temperature increases above the lattice temperature, so the scattering rate increases. The variation of the scattering rate with (lattice) temperature is investigated more fully in Fig. 3, where the expected fall off with temperature is apparent (as the temperature decreases, the lattice vibrations become smaller and so we expect less scattering). As can be noted from the figure, the drop off in scattering rate with temperature is quite marked, becoming negligible as the temperature falls below approximately $150 \mathrm{~K}$.

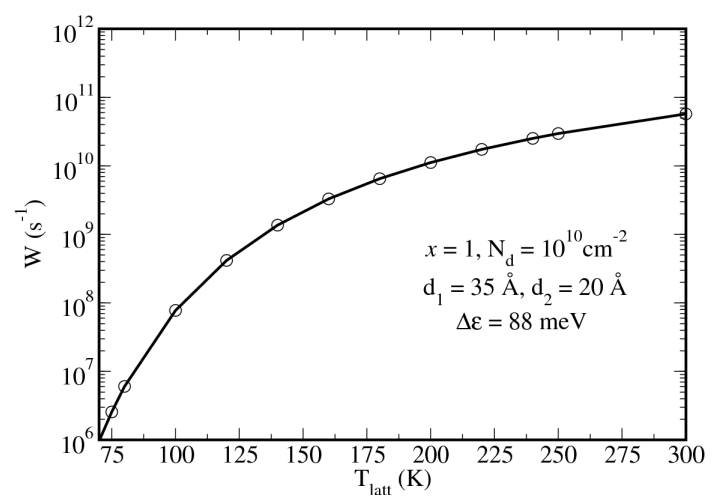

Fig. 3. The variation of the scattering rate with lattice temperature. The fall off below lattice temperatures of $150 \mathrm{~K}$ is quite marked. 
The results for the variation of scattering rate with doping density, $N_{\mathrm{d}}$, are presented in Fig. 4. We note an almost linear dependence with doping density for $T_{\text {latt }}=300 \mathrm{~K}$, while that for the lower lattice temperatures has more structure. This variation can be explained by noting that the Fermi energy, $\varepsilon_{\mathrm{F}}$, depends upon the doping density, $N_{\mathrm{d}}$, in the following manner (assuming one bound $\Gamma$ state): $\varepsilon_{\mathrm{F}}=\varepsilon_{\mathrm{F}}(0)+k_{\mathrm{B}} T \ln \left(1-\exp \left(-2 \pi B N_{\mathrm{d}}\right)\right)$ [4] with $\varepsilon_{\mathrm{F}}(0)$ given as $E_{n}^{\Gamma}+$ $2 \pi \hbar^{2} N_{\mathrm{d}} / 2 m^{\Gamma}$ and $B=\beta \hbar^{2} / 2 m^{\Gamma}$. Hence, for large temperatures the exponential term becomes small and there is an almost linear dependence on the Fermi energy with doping density, while for smaller lattice temperatures the dependence is more complicated. Considering the expression for the scattering rate, it can be shown that we expect dependence between the energy difference between the $\Gamma$-level and the Fermi energy.

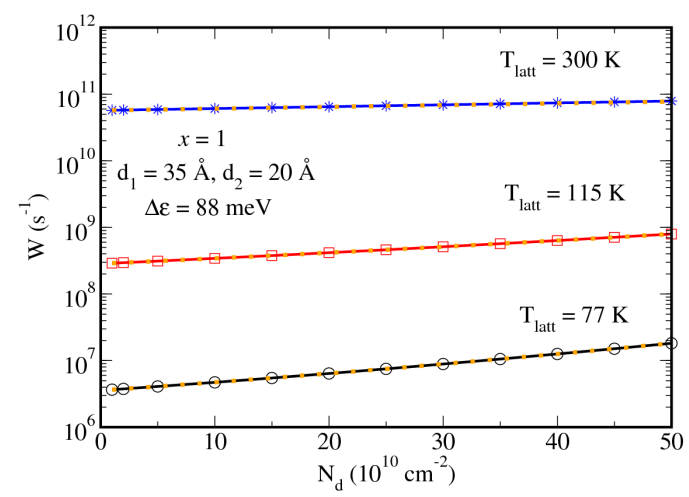

Fig. 4. The variation of the scattering rate with doping density, $N_{\mathrm{d}}$. The full curves correspond to ignoring Pauli blocking. As can be noticed, the Pauli blocking is negligible for the range of doping densities considered.

This linear dependence of scattering rate with doping density will, of course, eventually drop off, due to the Pauli blocking in the final state. To check this, we have plotted also in Fig. 4 the results of including Pauli blocking, assuming the $\Gamma$ - and $X$-states are in thermal equilibrium. As can be noted, for the range of carrier densities considered, the results of including Pauli blocking is negligible and ignoring it is reasonable.

We can show that we expect the Pauli blocking to be small when the following inequality is (approximately) satisfied:

$$
2 \pi^{2} \frac{\left(\hbar^{2} N_{\mathrm{d}} / 2 m\right)^{2}}{k_{\mathrm{B}} T_{\mathrm{e}}} \ll E_{n^{\prime}}^{X}-E_{n}^{\Gamma},
$$

which is true for the doping densities and temperatures considered.

We now discuss the variation of the scattering rate with applied electric field. These results are presented in Fig. 5 where we note, for the range of fields considered, an almost linear relationship between scattering rate and applied field for 
large lattice temperatures, but which breaks down for lower lattice temperatures. We can explain this variation by noting that, because the widths of the two wells are different, when we apply an electric field the energies of the $\Gamma$ - and $X$-levels change by different amounts. This means that the difference in energies will vary and (making use of the results presented in Fig. 1) we know that as this energy difference varies then so does the scattering rate.

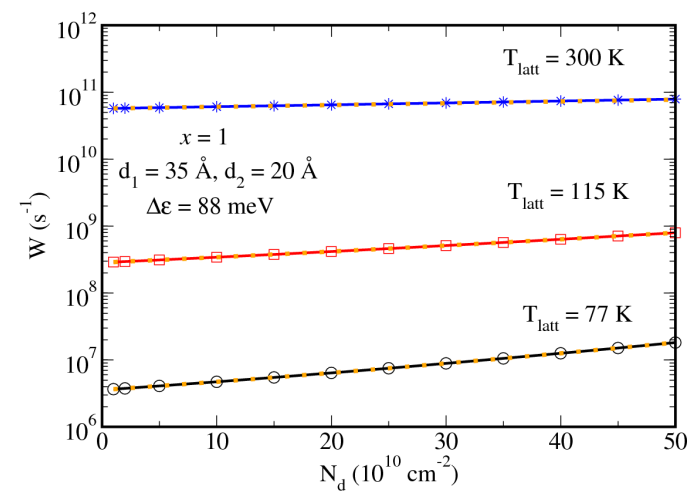

Fig. 5. The variation of the scattering rate with applied electric field. As the field changes so do the energies of the $\Gamma$ - and $X$-states. The changes in energies for the $\Gamma$ and $X$-states are different because of the different widths of each well.

Before discussing a realistic device, we consider a double well, see Fig. 6 . In this figure we compare the scattering rate from the lowest bound state in the left hand well to the right hand well, $W_{\Gamma_{\mathrm{R}} \Gamma_{\mathrm{L}}}$, with the scattering rates $W_{\Gamma_{\mathrm{R}} \mathrm{X}}$ and $W_{X \Gamma_{\mathrm{L}}}$, where $X$ represents all the $X$-states present. From Fig. 6, we note then that the two scattering processes are comparable.

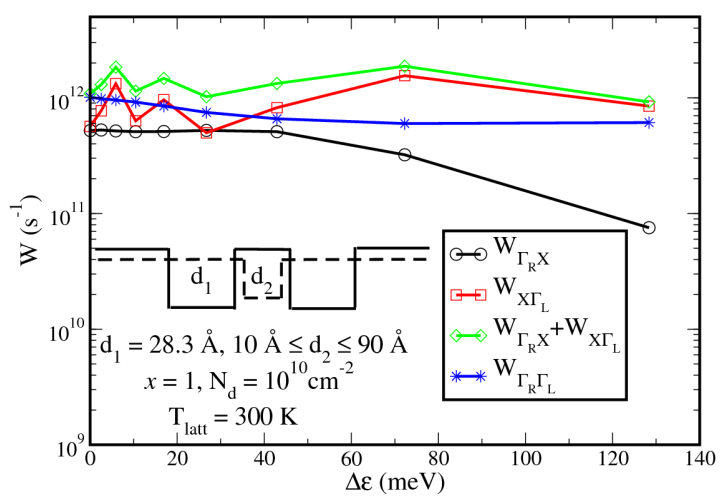

Fig. 6. The scattering rate versus energy for a double quantum well. The inset shows the $\Gamma$ (full line) and $X$ (dashed line) potential profiles. Let us note the much less rapid fall off of the scattering rate with energy when compared with Fig. 1. 
The structure found in the $X \Gamma_{\mathrm{L}}$ data (and to a lesser extent in the $\Gamma_{\mathrm{R}} X$ data) can be explained by noting that as the width of the $X$-well increases, more $X$-states are present. The much less rapid fall off with energy compared with the results for one well may be accounted for by noting that compared to the results for one well, the $\Gamma$-states extend to a greater amount into the barrier region, so that the overlap integrals are correspondingly larger in this case.

It is noteworthy to point out the importance of including the $X$-states in determining the Fermi energy. This may be surprising at first that the $X$-states will have such a large effect, but this can be defended by noting that effective mass of the electron in the $X$-well is some twelve times larger than in the $\Gamma$-well.

Finally, we compare results with the work of Page et al. [18], who reported on the operation of a $\mathrm{GaAs} / \mathrm{Al}_{0.45} \mathrm{Ga}_{0.55} \mathrm{As}$ based QCL at $\lambda \approx 9 \mu \mathrm{m}$ for a working field of $48 \mathrm{kV} / \mathrm{cm}$ and lattice temperature of $300 \mathrm{~K}$. Two periods of the device are shown in Fig. 7, with the $\Gamma$-states indicated. Also shown are the $X$ potential profile and the corresponding $X$-states. We determine the self-consistent scattering rates, accounting for all electron-longitudinal-optical phonon and electronelectron scattering rates as well as an evaluation of the non-equilibrium electron distribution $[34,35]$.

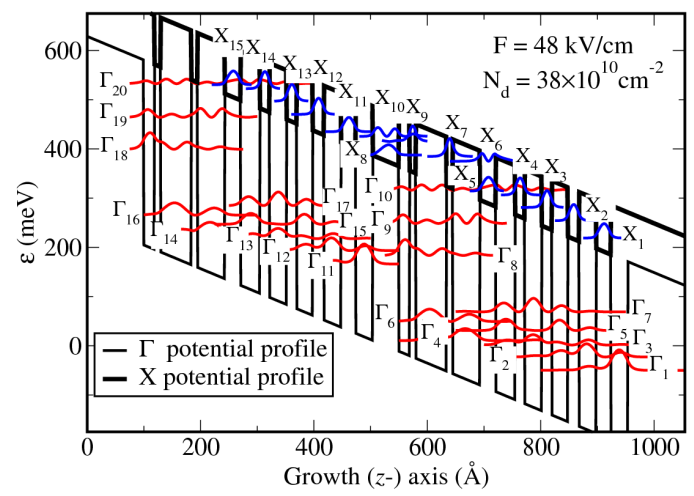

Fig. 7. The $\Gamma$ and $X$ potential profiles with the relevant wave functions used to determine the scattering rate.

Table I shows the results for our scattering rate determination for $W_{8}, W_{9}$, and $W_{10}$, where $W_{i}$ is the scattering rate from the range of states $\Gamma_{11}-\Gamma_{20}$ to the state $\Gamma_{i}$, representing the transition rate from the left hand period to those selected states of interest in the right hand period. These states are the upper and lower laser levels, $\Gamma_{9}$ and $\Gamma_{8}$, respectively, and a continuum state, $\Gamma_{10}$, discussed by Indjin et al. [36] in their analysis of the device of Page et al. [18]. We note these scattering rates are of order $0.1-1 \times 10^{12} \mathrm{~s}^{-1}$.

To determine an estimate of the contribution from $\Gamma \rightarrow X$ scattering we only consider those $\Gamma$ and $X$-states indicated in the figure, and apply the results 
TABLE I

Scattering rates for the range of states $\Gamma_{11}-\Gamma_{20}$ to the state $\Gamma_{i}$.

\begin{tabular}{c|c}
\hline \hline$\Gamma_{i}$ & $W_{i}\left[\mathrm{~s}^{-1}\right]$ \\
\hline$\Gamma_{8}$ & $5.98 \times 10^{11}$ \\
$\Gamma_{9}$ & $7.95 \times 10^{11}$ \\
$\Gamma_{10}$ & $1.11 \times 10^{11}$
\end{tabular}

TABLE II

Scattering rates for the state $\Gamma_{i}$ to the state $X_{5}$.

\begin{tabular}{c|c}
\hline \hline$\Gamma_{i}$ & $W_{i}\left[\mathrm{~s}^{-1}\right]$ \\
\hline$\Gamma_{8}$ & $7.21 \times 10^{9}$ \\
$\Gamma_{9}$ & $3.55 \times 10^{11}$ \\
$\Gamma_{10}$ & $2.76 \times 10^{12}$
\end{tabular}

\section{TABLE III}

Scattering rates for the range of states $\Gamma_{11}-\Gamma_{20}$ to the state $X_{i}$.

\begin{tabular}{c|c}
\hline \hline$X_{i}$ & $W_{i}\left[\mathrm{~s}^{-1}\right]$ \\
\hline$X_{1}$ & $3.85 \times 10^{11}$ \\
$X_{2}$ & $4.31 \times 10^{11}$ \\
$X_{3}$ & $2.29 \times 10^{11}$ \\
$X_{4}$ & $2.40 \times 10^{11}$ \\
$X_{5}$ & $2.85 \times 10^{11}$
\end{tabular}

for the scattering rate discussed previously. We point out two approximations that we make: (i) Raichev's result was derived for a superlattice and strictly does not apply to the structure designed by Page et al.; (ii) Because the $\Gamma$-states can be appreciable over several quantum wells, we suppose them to belong to an "effective quantum-well". Our results are presented in Tables II and III. Table II shows the results of our calculations for considering the scattering rates from the $\Gamma$-states $\Gamma_{8}, \Gamma_{9}$ and $\Gamma_{10}$ to the $X$-state $X_{5}$ (this state is expected to have the largest contribution because of its energy and position with respect to the states under consideration) for a lattice temperature of $300 \mathrm{~K}$ and a doping density of $38 \times 10^{10} \mathrm{~cm}^{-2}$.

We note that as the temperature decreases the corresponding scattering rate falls of drastically, for example for the transition $\Gamma_{9} \rightarrow X_{5}$, the scattering drops to $3.76 \times 10^{5} \mathrm{~s}^{-1}$ for $T_{\text {latt }}=115 \mathrm{~K}$ and $9.50 \times 10^{0} \mathrm{~s}^{-1}$ for $77 \mathrm{~K}$. 
Finally, Table III shows the results for the scattering rates from the $\Gamma$-states $\Gamma_{11-20}$ to the different $X$-states. The reason for this being these states, although less spatially closer to each other, they are energetically close. We note that the relative importance of the two scattering processes can vary quite dramatically depending upon the transition involved.

Comparing Tables II and III with Table I we see that it could be important to take into account the effects of $\Gamma-X$ scattering [26] for the device of Page et al. under consideration, though we do note these calculations are not self-consistent.

\section{Conclusions}

Our calculations of inter-valley scattering suggest that $\Gamma-X$ scattering can be as fast as acoustic phonon scattering, particularly when the $X$-states are close in energy to those originating from the $\Gamma$-valley as can happen when the $\mathrm{Al}$ concentration in the AlGaAs alloy approaches $45 \%$.

\section{Acknowledgments}

This work was supported in part by the EPSRC (U.K.) grant reference number GR/C002881/1.

\section{References}

[1] R. Tsu, L. Esaki, Appl. Phys. Lett. 22, 562 (1973).

[2] L.L. Chang, L. Esaki, R. Tsu, Appl. Phys. Lett. 24, 593 (1974).

[3] T.C.L. G. Sollner, W.D. Goodhue, P.E. Tannenwald, C.D. Parker, D.D. Beck, Appl. Phys. Lett. 43, 588 (1983).

[4] T.J. Shewchuk, P.C. Chapin, P.D. Coleman, W. Kopp, R. Fischer, H. Morko, Appl. Phys. Lett. 46, 508 (1985).

[5] M. Tsuchiya, H. Sakaki, Y. Joshino, Jpn. J. Appl. Phys. 24, L466 (1985).

[6] V.Ya. Aleshkin, L. Reggiani, M. Rosini, Phys. Rev. B 73, 165320 (2006), and references therein.

[7] E.E. Mendez, E. Calleja, C.E.T. Gonalves da Silva, L.L. Chang, W.I. Wang, Phys. Rev. B 33, 7368 (1986).

[8] E.E. Mendez, W.I. Wang, E. Calleja, C.E.T. Gonalves da Silva, Appl. Phys. Lett. 50, 1263 (1987).

[9] A.C. Marsh, Semicond. Sci. Technol. 1, 320 (1986).

[10] H.C. Liu, Appl. Phys. Lett. 51, 1019 (1987).

[11] J. Faist, F. Capasso, D.L. Sivco, C. Sirtori, A.L. Hutchinson, A.Y. Cho, Science 264, 553 (1994).

[12] F. Capasso, C. Gmachl, D.L. Sivco, A. Y. Cho, Physics Today 55, 34 (2002).

[13] O. Malis, C. Gmachl, D.L. Sivco, L.N. Pfeiffer, A.M. Sergent, K.W. West, Bell Labs Techn. J. 10, 199 (2005).

[14] C. Sirtori, H. Page, C. Becker, Philos. Trans. R. Soc. Lond. A 359, 505 (2001). 
[15] C. Sirtori, J. Nagle, C.R. Physique 4, 639 (2003).

[16] C. Sirtori, P. Kruck, S. Barbieri, P. Collot, J. Nagle, M. Beck, J. Faist, U. Oesterle, Appl. Phys Lett. 73, 3486 (1998).

[17] C. Sirtori, P. Kruck, S. Barbieri, H. Page, J. Nagle, Appl. Phys. Lett. 75, 3911 (1999).

[18] H. Page, C. Becker, A. Robertson, G. Glastre, V. Ortiz, C. Sirtori, Appl. Phys. Lett. 78, 3529 (2001).

[19] R. Kohler, A. Trediccuci, F. Beltram, H.E. Beere, E.H. Linfield, A.G. Davies, D.A. Ritchie, R.C. Iotti, F. Rossi, Nature 417, 156 (2002).

[20] J.S. Roberts, R.P. Green, L.R. Wilson, E.A. Zibik, D.G. Revin, J.W. Cockburn, R.J. Airey, Appl. Phys. Lett. 82, 4221 (2003).

[21] M. Garcia, E. Normand, C.R. Stanley, C.N. Ironside, C.D. Farmer, G. Duxbury, N. Langford, Opt. Commun. 226, 39 (2003).

[22] J.-Y. Bengloan, A. De Rossi, V. Ortiz, X. Marcadet, M. Calligaro, I. Maurin, C. Sirtori, Appl. Phys. Lett. 84, 2019 (2004).

[23] R. Maulini, A. Mohan, M. Giovannini, J. Faist, E. Gini, Appl. Phys. Lett. 88, 1 (2006).

[24] S. Adachi, J. Appl. Phys. 58, R1 (1985).

[25] I. Vurgaftman, J.R. Meyer, L.R. Ram-Mohan, J. Appl. Phys. 89, 5815 (2001).

[26] X. Gao, B. Bortez, J. Knezević, Appl. Phys. Lett. 89, 191119 (2006); J. Appl. Phys. 101, 063101 (2007).

[27] L.R. Wilson, D.A. Carder, J.W. Cockburn, R.P. Green, D.G. Revin, M.J. Steer, M. Hopkinson, G. Hill, R. Airey, Appl. Phys. Lett. 81, 378 (2002).

[28] R. Tessier, J.J. Finley, M.S. Skolnick, J.W. Cockburn, J.-L. Pelouard, R. Grey, G. Hill, M.A. Pate, R. Planel, Phys. Rev. B 54, R8329 (1996).

[29] R. Diniz, J. Smoliner, E. Gornik, U. Meiners, H. Brugger, P. Wisniewski, T. Suski, Semicond. Sci. Technol. 8, 1352 (1993).

[30] S.R. Jin, C.N. Ahmad, S.J. Sweeney, A.R. Adams, B.N. Murdin, H. Page, X. Marcadet, C. Sirtori, S. Tomić, Appl. Phys. Lett. 89, 221105 (2006).

[31] O.E. Raichev, Phys. Rev. B 49, 5448 (1994).

[32] G.A. Bastard, Wave mechanics applied to semiconductor structures, Les Editions de Physique, Les Ulis 1988.

[33] P. Harrison, Quantum Wells, Wires and Dots: Theoretical and Computational Physics, Wiley, Chichester 2005.

[34] P. Harrison, D. Indjin, Z. Ikonić, V.D. Jovanović, J. Mc Tavish, R.W. Kelsall, I. Savić, in: 12th Int. Symp. Nanostructures: Physics and Technology, Eds. Zh. Alferov, L. Esaki, Ioffe Inst., St. Petersburg 2004, p. 79, and references therein.

[35] J.P. Mc Tavish, D. Indjin, P. Harrison, J. Appl. Phys. 99, 103106 (2006), and references therein.

[36] D. Indjin, P. Harrison, R.W. Kelsall, Z. Ikonić, Appl. Phys. Lett. 81, 400 (2002). 